\title{
A Numerical Study on the Validity of the Local Equilibrium Hypothesis in Modeling Hydrogen Thermal Desorption Spectra
}

\author{
Ken-ichi EBIHARA, ${ }^{1)}$ Hideo KABURAKI, ${ }^{1)}$ Tomoaki SUZUDO ${ }^{1)}$ and Kenichi TAKAI ${ }^{2)}$ \\ 1) Center for Computational Science \& e-Systems, Japan Atomic Energy Agency, Tokai-mura, Naka-gun, Ibaraki $319-1195$ \\ Japan. $\quad 2$ 2) Department of Engineering and Applied Science, Sophia University, Kioi-cho, Chiyoda-ku, Tokyo $102-8554$
} Japan.

(Received on July 27, 2009; accepted on August 14, 2009)

\begin{abstract}
We present a systematic benchmark study on different numerical models for analyzing hydrogen thermal desorption spectra, by focusing on the adoption of the local equilibrium hypothesis in these models. We find that the direct numerical method of the full set of the extended mass conservation equations is only able to predict the experimental behavior of thermal desorption spectra for pure iron in the thin specimen limit, while other models incorporating the local equilibrium hypothesis fail to predict this behavior.
\end{abstract}

KEY WORDS: thermal desorption analysis; hydrogen state; local equilibrium; hydrogen diffusion; numerical simulation.

\section{Introduction}

Hydrogen in steels affects significantly their mechanical properties, and, in particular, hydrogen-induced cracking is a problem for the welding process of high-strength steels. Hydrogen atoms in steels are located mostly at trapping defect sites such as vacancies, dislocations, grain boundaries, and phase interfaces. The understanding of these trapping hydrogen states is especially required to predict a delayed fracture process, because hydrogen atoms diffuse in a complex way by being captured by and released from trapping sites depending on the stress and temperature conditions.

Each trap site has its own characteristic binding energy, corresponding to the temperature at which hydrogen is released from the trapping site potential. Thus, thermal desorption analysis (TDA) ${ }^{1,2)}$ is the most useful experimental method for identifying a hydrogen state in steels to delve into such trapping properties of defects. A hydrogen desorption profile is obtained through TDA by measuring an amount of hydrogen released from a specimen heated at a constant rate. The amount of hydrogen trapped at each defect is evaluated from the thermal desorption profile because the type of defect can be identified from the temperature of the hydrogen desorption peak in the spectra. Recently, the low-temperature TDA technique has been developed, ${ }^{3)}$ and we can obtain detailed spectra that include the desorption peaks corresponding to defects whose binding energies are comparatively low such as grain boundaries, dislocations, and vacancies. Since binding energies of these defects are close to each other, corresponding hydrogen desorption peaks overlap where it is generally difficult in experiment to separate the amount of hydrogen trapped at each type of defects. Thus, numerical modeling of this thermal desorption process is particularly important for analyz- ing the spectra.

A theoretical study for modeling thermal desorption spectra in steels dates back to the paper by McNabb and Foster $^{4}$ in 1963. In their paper, they proposed the diffusion equation incorporating the effect of hydrogen states in the lattice, namely capturing and releasing process of hydrogen at defect sites, in order to explain anomalous hydrogen diffusion process, in which the values of hydrogen diffusion coefficient in the lower temperature region scatter widely depending on the work hardening process of the specimen. In 1970, Oriani ${ }^{5}$ ) reformulated the equation by $\mathrm{McNabb}$ and Foster, introducing the local equilibrium postulate of hydrogen between trap sites and interstitial lattice sites. The interstitial lattice site is referred as the free space in the lattice where hydrogen diffusion occurs. He proposed the effective diffusion coefficient which includes the effect of trapping hydrogen at defect sites. He also considered the hydrogen state for the explanation of hydrogen diffusion anomaly. Furthermore, the hydrogen state, such as the hydrogen trapping effect by defects, was studied in more detail ${ }^{6)}$ in order to explain the anomaly of hydrogen diffusion. However, the study of a diffusion coefficient is unsuitable for identifying the hydrogen state which is determined by various factors, such as the temperature, the binding energy, the density of trapping defect sites, etc. On the other hand, the method of thermal desorption under controlled temperature-increasing conditions is used for identifying the hydrogen state. For this analysis, capture and release of hydrogen for a trap site is modeled as an activation process. Thus, in order to explain the thermal desorption spectra, the activation process of trapping and detrapping and the diffusion process are combined in the mass conservation equation for a single type of $\operatorname{trap}^{7)}$ and two types of traps. ${ }^{8)}$ The formulation used in these works is similar to that of McNabb and Foster. 
There is also a work in which only the releasing process of hydrogen is taken into account to reproduce the desorption profile. ${ }^{9,10)}$ The local equilibrium hypothesis is employed in some works. ${ }^{11,12)}$ The objective of these works is the explanation of the thermal desorption spectra using the diffusion and activation processes or the local equilibrium hypothesis.

Recently, numerical simulations are performed to study the entire temperature range of the hydrogen desorption spectra, and the performance of the model is examined by studying the sensitivity to several simulation parameters. ${ }^{13-15)}$ Turnbull et al. ${ }^{13)}$ proposed a rigorous model for simulating the profile with two desorption peaks on the basis of the work of Wilson and Baskes. ${ }^{7}$ The proposed model has the mass conservation form of hydrogen with two trap-site terms described by the activation energy. This set of equations is nonlinear and includes time-dependent hydrogen concentration terms at interstitial lattice sites and trap sites. The effect on the peak location of desorption spectra is studied by changing the trap site density in a carbon steel. Enomoto et al., ${ }^{14)}$ using a model similar to that of Turnbull et al., studied the dependence of the desorption spectra for two kinds of carbon steels on the heating rate and the exposure time before heating. Yamaguchi and Nagumo ${ }^{15)}$ used the model on the basis of the one-dimensional diffusion equation with the effective diffusion coefficient that is deduced by Oriani ${ }^{5)}$ using the local equilibrium hypothesis, and simulated the dependence of the deformation rate, the heating rate, and the exposure time on the desorption spectra. Ebihara et al. ${ }^{16)}$ proposed a simple non-diffusion model on the assumption that hydrogen diffuses extremely fast in steels, ${ }^{9,10)}$ where the local equilibrium hypothesis is also adopted to remove the time dependent trap site terms: The numerical results are compared with the experimental thermal desorption spectra of pure iron and eutectoid steel using bulk specimens, and are successful in reproducing the location and the relative decrease of the desorption peaks in the profiles in the entire temperature range.

As described above, although a variety of models is proposed to simulate the hydrogen thermal desorption spectra, a systematic study on the sensitivity of parameters and the validity of the approximations and assumptions in the models has not been made, due partly to the lack of comprehensive experimental data for modeling. With the recent advancement of thermal desorption measurement from the low temperature, ${ }^{3)}$ thermal desorption spectra of hydrogen for pure iron are obtained over a wide temperature range, which gives an impetus to the validation of the numerical models.

This paper is organized as follows. The next section introduces three models used in this paper for comparative studies and simulation conditions. The simulation results of each model are shown in the Sec. 3 and they are discussed in the Sec. 4. The final section summarizes the paper.

\section{Numerical Models and Conditions}

In this section, we briefly introduce three models for simulating thermal desorption spectra and simulation conditions for comparative studies.

\subsection{Simulation Models}

Firstly, we introduce a one-dimensional time-dependent mass conservation model that includes diffusion of hydrogen and rates of all capture and release processes in the lattice. This model is originally presented by Wilson and Baskes, ${ }^{7)}$ extended by Turnbull et al., ${ }^{13)}$ and used by Enomoto et al. ${ }^{14)}$ We call this model Model I for simplicity. The main equations of Model I in the cylindrical coordinates are as follows:

$$
\begin{gathered}
\frac{\partial C(x)}{\partial t}+\sum_{i} N_{i} \frac{\partial \theta_{i}(x)}{\partial t}=D \frac{\partial^{2} C(x)}{\partial x^{2}}+D \frac{1}{x} \frac{\partial C(x)}{\partial x} \\
\frac{\partial \theta_{i}(x)}{\partial t}=k_{i}(T) C(x)\left(1-\theta_{i}(x)\right)-p_{i}(T) \theta_{i}(x) \ldots \ldots
\end{gathered}
$$

where $x[\mathrm{~m}]$ is a distance from the axis of the cylindrical specimen. Here $C(x)\left[/ \mathrm{m}^{3}\right]$ is the amount of hydrogen in an interstitial lattice site at the position $x$, through which hydrogen migrates. The amount of hydrogen in the trap site of $i$ type is represented by the product of trap site concentration $N_{i}\left[/ \mathrm{m}^{3}\right]$ and the occupation ratio $\theta_{i}(x)$ that is dimensionless and takes 1.0 when all the trap sites of $i$ type is completely occupied by hydrogen. Time and temperature are $t[\mathrm{~s}]$ and $T[\mathrm{~K}]$, respectively. The rate of capturing and releasing hydrogen by trap sites and the diffusion coefficient are $k_{i}\left[\mathrm{~m}^{3} / \mathrm{s}\right], p_{i}[/ \mathrm{s}]$, and $D\left[\mathrm{~m}^{2} / \mathrm{s}\right]$, respectively, and they follow:

$$
\begin{gathered}
k_{i}=k_{0} \exp \left(\frac{-Q_{D}-E_{i}^{\prime}}{R T}\right) \ldots \ldots \\
p_{i}=p_{0} \exp \left(\frac{-Q_{D}-E_{i}-E_{i}^{\prime}}{R T}\right) \\
D=D_{0} \exp \left(-\frac{Q_{D}}{R T}\right) \ldots \ldots \ldots
\end{gathered}
$$

Here $Q_{D}[\mathrm{~kJ} / \mathrm{mol}], E_{i}[\mathrm{~kJ} / \mathrm{mol}]$, and $E_{i}^{\prime}[\mathrm{kJ} / \mathrm{mol}]$ are hydrogen activation energy, binding energy, and barrier energy, respectively. The gas constant is $R=8.31 \mathrm{~J} / \mathrm{mol} \cdot \mathrm{K}$ and the pre-exponential factor is represented by $k_{0}, p_{0}$, and $D_{0}$, respectively. The relation $p_{0} / k_{0}=N_{I},{ }^{13)}$ where $N_{I}\left[/ \mathrm{m}^{3}\right]$ is the concentration of interstitial lattice sites, is assumed in this model.

The model based on the one-dimensional diffusion equation incorporating the local equilibrium hypothesis is firstly used by Yamaguchi and Nagumo ${ }^{15)}$ to study the sensitivity of the hydrogen desorption spectra to several parameters such as binding energy and trap site density. This model explicitly includes the local equilibrium hypothesis. We hereafter call this model Model II. The diffusion equation of Model II in the cylindrical coordinates is described as follows:

$$
\frac{\partial C(x)}{\partial t}=\hat{D} \frac{\partial^{2} C(x)}{\partial x^{2}}+\hat{D} \frac{1}{x} \frac{\partial C(x)}{\partial x}
$$




$$
\hat{D}=D_{0} \exp \left(\frac{-Q_{D}}{R T}\right)\left[1+\sum_{i} K_{i} \exp \left(\frac{E_{i}}{R T}\right)\right]^{-1}
$$

Here $K_{i}$ is the ratio of trap site density to interstitial lattice site density, $N_{i} / N_{I}$. Equation (6) is an ordinary diffusion equation with the effective diffusion coefficient renormalized to include the effect of trapping hydrogen. ${ }^{5)}$

The third model, Model III, is constructed by adding the diffusion process into the non-diffusion model. This non-diffusion model, originally proposed by the present authors, ${ }^{16)}$ is basically the continuity of mass equation with hydrogen trap sites in the activation energy form (Fig. 1). Here the diffusion of hydrogen is assumed to be fast and is neglected. Instead the local equilibrium hypothesis is imposed at each step in the temperature raising process, and hydrogen in the interstitial lattice site is directly desorbed. Model III is constructed by aligning the non-diffusion model in one-dimensional direction as shown in Fig. 2. A non-diffusion model at each site in the one dimensional direction $x$ is termed a "cell" in this figure. Hydrogen diffusion in the interstitial lattice sites is calculated by the ordinary diffusion equation as follows:

$$
\frac{\partial C(x)}{\partial t}=D \frac{\partial^{2} C(x)}{\partial x^{2}}+D \frac{1}{x} \frac{\partial C(x)}{\partial x}
$$

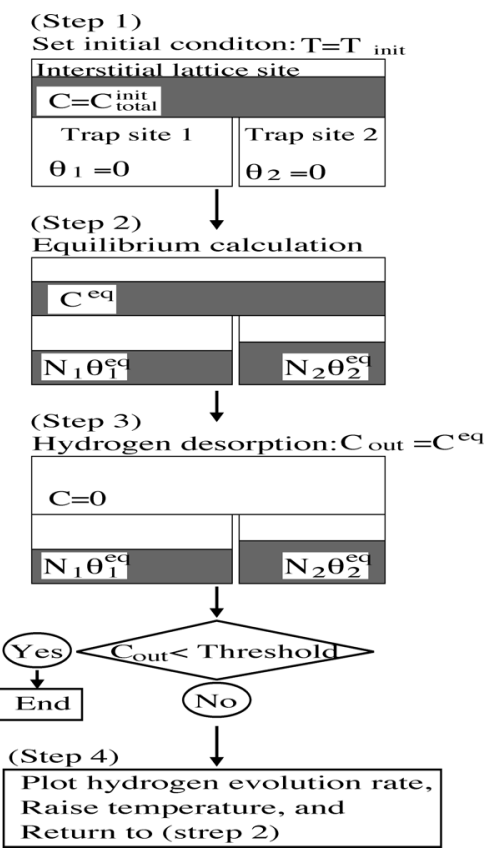

Fig. 1. Non-diffusion model: This illustrates the case that there are two types of trapping sites. (Step 1) The initial conditions are set; $C=C_{\text {total }}^{\text {init }}, T=T_{\text {init, }}, \theta_{1}=\theta_{2}=0$. The dark hatching area at each site represents the hydrogen concentration. (Step 2) By the equilibrium calculation, the amount of hydrogen at the interstitial lattice site, $C^{\mathrm{eq}}$, and at trapping sites, $N_{1} \theta_{1}^{\text {eq }}, N_{2} \theta_{2}^{\text {eq }}$, is obtained. (Step 3) Hydrogen in the interstitial lattice site is removed as the desorbed hydrogen; $C_{\text {out }}=C^{\text {eq }}$ and the amount of hydrogen at the interstitial lattice site is set at zero. (Step4) The hydrogen evolution rate is obtained from $C_{\text {out }}$, and the temperature is increased by $\Delta T=$ [heating rate $\times d t]$. Then (Step 2) is applied to the hydrogen state of (Step 3) with the new temperature.

$$
D=D_{0} \exp \left(\frac{-Q_{D}}{R T}\right)
$$

Here $D$ is the intrinsic diffusion coefficient of hydrogen in the lattice and is different from the renormalized value in Eq. (7). The procedure for obtaining the desorption profile is shown also in Fig. 2.

\subsection{Simulation Conditions}

The following simulation conditions are specified for a benchmark of the above three models. We consider a desorption process of the cylindrical pure iron specimen annealed at $873 \mathrm{~K}$ after $83 \%$ deformation because the microstructure of the specimen is comparatively simple. This condition is also used in our previous paper. ${ }^{16)}$ The parameters in the simulation condition are set as follows: $E_{d}=58.6 \mathrm{~kJ} / \mathrm{mol}, Q_{D}=8.0 \mathrm{~kJ} / \mathrm{mol}, E^{\prime}=0, N_{d}=3.48 \times$ $10^{26} / \mathrm{m}^{3}, \quad N_{I}=7.61 \times 10^{29} / \mathrm{m}^{3}, \quad k_{0}=1.0 \times 10^{-26} \mathrm{~m}^{3} / \mathrm{s}$, heating rate $V_{T}=200 \mathrm{~K} / \mathrm{h}$, initially charged hydrogen $C_{\text {total }}^{\text {init }}=4.8$ mass ppm, $D_{0}=4.4 \times 10^{-8} \mathrm{~m}^{2} / \mathrm{s}$, the radius of the cylindrical specimen $L=2.5 \times 10^{-3} \mathrm{~m}$, the spacing of discretization

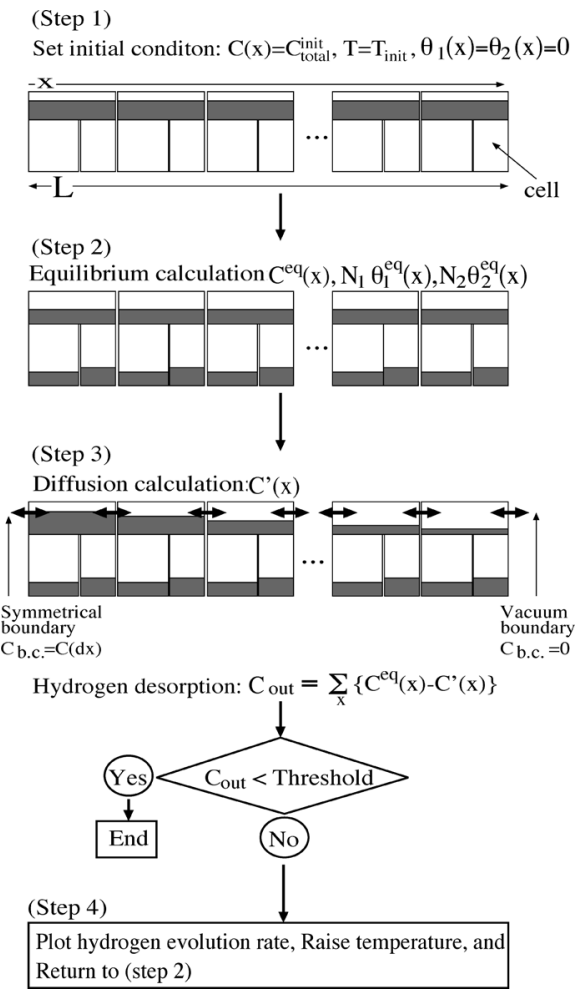

Fig. 2. Model III: This model is constructed by aligning the cells in a one-dimensional direction, i.e. there is one cell at each discretized point in the $x$ direction. (Step 1) The initial conditions are set; $C(x)=C_{\text {total }}^{\text {init }}, T=T_{\text {init }}, \theta_{1}(x)=$ $\theta_{2}(x)=0$. The dark hatching area at each site represents the hydrogen concentration. (Step 2) By the equilibrium calculation, the amount of hydrogen at the interstitial lattice site, $C^{\mathrm{eq}}(x)$, and at trapping sites, $N_{1} \theta_{1}^{\mathrm{eq}}(x), N_{2} \theta_{2}^{\mathrm{eq}}(x)$, are obtained at each cell. (Step 3) By calculating diffusion of hydrogen, hydrogen at the interstitial lattice site, $C^{\prime}(x)$, is obtained for each cell. Then the desorbed hydrogen is obtained as $C_{\text {out }}=\sum_{x}\left\{C^{\mathrm{eq}}(x)-C^{\prime}(x)\right\}$. (Step 4) The hydrogen evolution rate is obtained from $C_{\text {out }}$, and the temperature is increased by $\Delta T=[$ heating rate $\times d t]$. Then (Step 2) is applied to the hydrogen state of (Step 3) with the new temperature. 


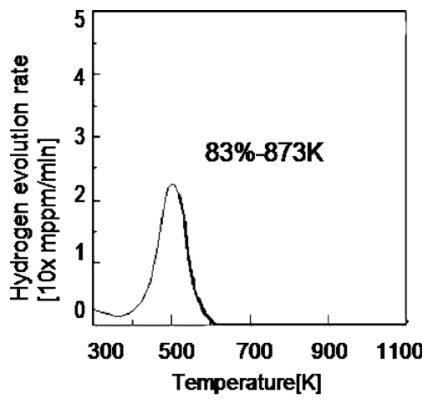

(a)

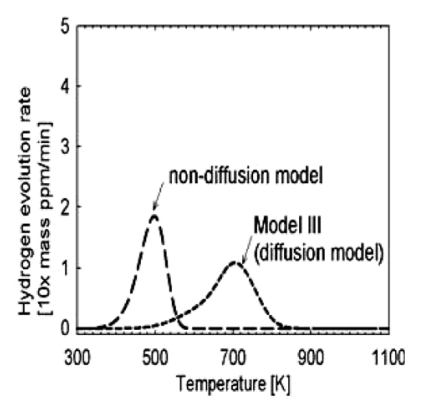

(b)

Fig. 3. Thermal desorption spectra for (a) the experiment ${ }^{1)}$ and (b) the simulation results by the non-diffusion model and Model III. ${ }^{16}$

grids $d x=2.5 \times 10^{-4} \mathrm{~m}$. The time step, $d t$, is determined so as to satisfy the condition of $D d t / d x^{2}=0.1$. The desorption process of hydrogen at interstitial lattice sites is modeled as the one-dimensional diffusion equation. ${ }^{17}$ ) The vacuum condition at the one end and the symmetric condition at the other end of the region are applied as the boundary conditions. In the simulation, dislocations are assumed as the only defect site for trapping hydrogen, because vacancies are all annealed out in the specimen. Figure 3 shows the desorption profiles obtained by the non-diffusion model and the Model III (diffusion model) under the same simulation condition. It is found that the non-diffusion model successfully simulates the experimental profile, while, on the other hand, the profile simulated by Model III shifts to the higher temperature. The behaviors of all three models I, II, III, and the non-diffusion model are compared under the same simulation condition with reference to experiment.

\section{Simulation Results}

With the three models, we studied the sensitivity of the desorption profile to several parameters. Firstly, the simulation results on the effects of the heating rate and the binding energy of the trap site are shown in Figs. 4 and 5, respectively. Here, the result of the non-diffusion model is shown for reference as an indication of the experimental profile. We can observe that the simulation results by the three models show similar behavior, indicating that these parameters are insensitive to the adoption of the equilibrium hypothesis. Besides, the variation of these parameters cannot reproduce the position and the width of the experimental peak profile.

Next we studied the sensitivity to the trap site density. The simulation results of the three models are shown in Fig. 6 as a function of the trap site density. In this figure,

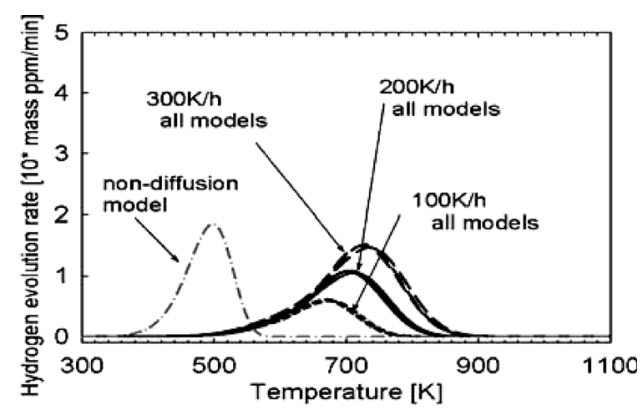

Fig. 4. Thermal desorption spectra by the three models as a function of the heating rate. The result of the non-diffusion model indicates the position of the profile in experiment

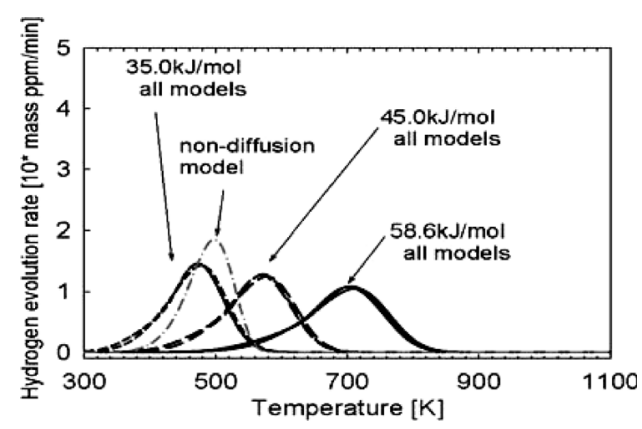

Fig. 5. Thermal desorption spectra by the three models as a function of the binding energy.

we can see that each model behaves differently as the trap site density decreases. In the case of Model I (Fig. 6(a)), the desorption peak shifts to the low temperature as the trap site density decreases, and comes to a halt around $600 \mathrm{~K}$. The position of this peak is still remote from that of experiment. Note that a new peak appears at the beginning of heating around $300 \mathrm{~K}$ in the smallest density. The peak of the desorption profile of Model II (Fig. 6(b)) moves to the lower temperature direction as the trap site density decreases and keeps shifting to the lower temperature indefinitely. It appears that the peak profile agrees with that of experiment, when the trap site density becomes small. Besides, the peak around $300 \mathrm{~K}$ never appears in this case. Model III is found to behave with both characteristics of Model I and II (Fig. 6(c)). We see that the peak around $300 \mathrm{~K}$, but the position of the peak continues to shift to the lower temperature as the trap site density decreases. Since the height of the peak decreases, the experimental profile cannot be reproduced with this model.

The effect of the specimen size on the desorption profile is shown in Fig. 7. In this case, Model II and Model III show almost identical behavior (Figs. 7(b), 7(c)), that is, the position of the profile continues to shift to the lower temperature direction and the peak steepens as the specimen size decreases. It is noticeable in Fig. 7(a) that the position of the peak profile for Model I stays around $600 \mathrm{~K}$ under a critical specimen size.

\section{Discussion}

\subsection{Comparison of Simulation Results}

In the previous section, we found that the trap site density and the specimen size cause a significant difference in 


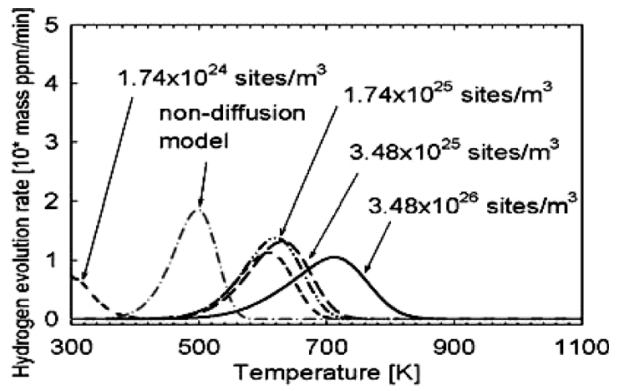

(a)

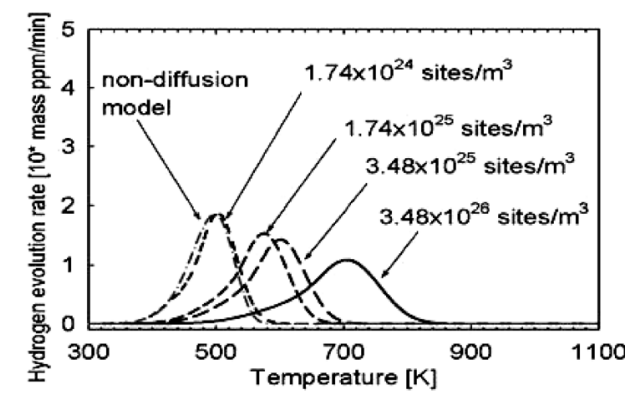

(b)

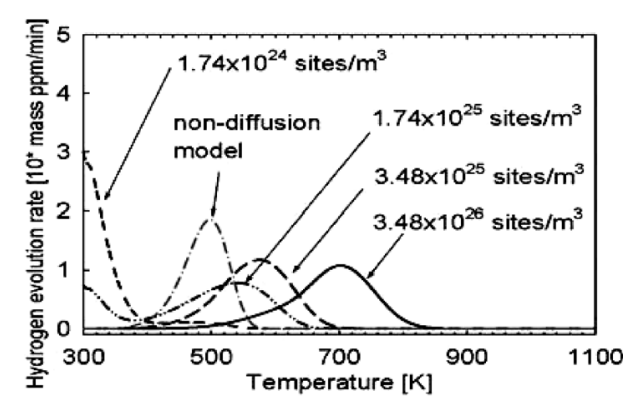

(c)

Fig. 6. Effects of the trap site density on the thermal desorption spectra by the three models: (a) Model I, (b) Model II, and (c) Model III.

the behavior of desorption profile among three models. As mentioned above, a major difference of Model I from the other models is that this model does not adopt directly the local equilibrium hypothesis. The other two models employ the local equilibrium hypothesis which is described mathematically as $\partial \theta / \partial t=0$ in Eq. (2). This relation is incorporated into the diffusion coefficient in Model II, while its constraint is enforced numerically at each temperature in Model III. Thus we can presume that the equilibrium of hydrogen fails in the process of decreasing the trap site density or the specimen size. To confirm this supposition for the case of Fig. 7, we calculate the ratio of the second term to the first term in the R.H.S. of Eq. (2), that is, $\left(N_{I} / C\right) \cdot\left\{\theta_{d} /\left(1-\theta_{d}\right)\right\} \cdot \exp \left(-E_{d} / R T\right)$ at $x=L$. Here the relation of $p_{0} / k_{0}=N_{I}$ is used. The result for Model I (Fig. 8(a)) shows that the ratio is almost unity when the specimen size is large, $L=2.5 \times 10^{-3} \mathrm{~m}$, and grows significantly as $L$ decreases. On the other hand, we see in Fig. 8(b) that the ratio for Model III remains to be almost unity and irrelevant to the length of $L$, which is obvious due to the numerically imposed condition of $\partial \theta / \partial t=0$. When $L$ is small for Model I, hydrogen quickly desorbs by diffusion, and, thus, the decrease of the amount of hydrogen $C$ in the first term of R.H.S. in Eq. (2) leads to the dominance of the second

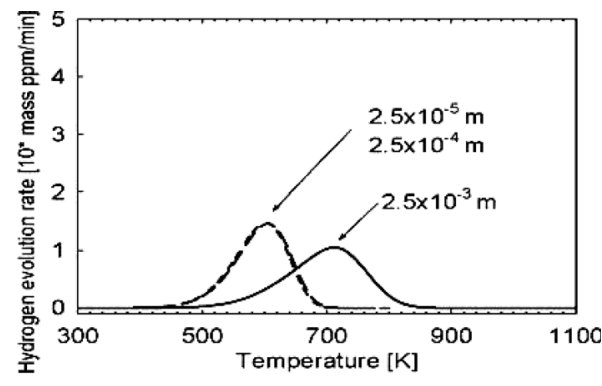

(a)

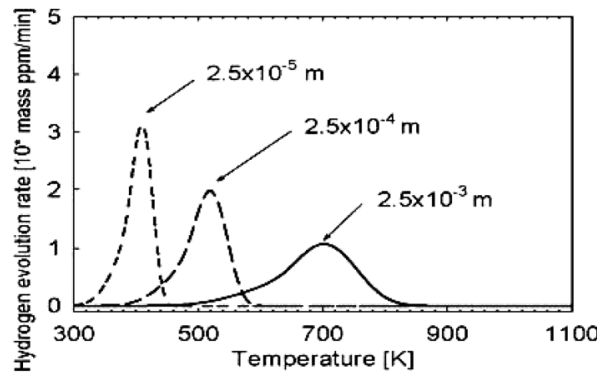

(b)

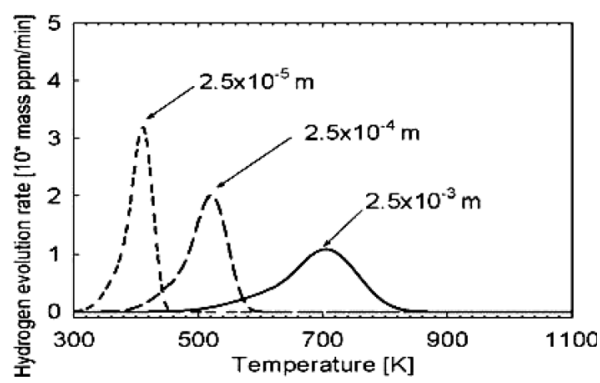

(c)

Fig. 7. Effects of the specimen size on the thermal desorption spectra by the three models: (a) Model I, (b) Model II, and (c) Model III.

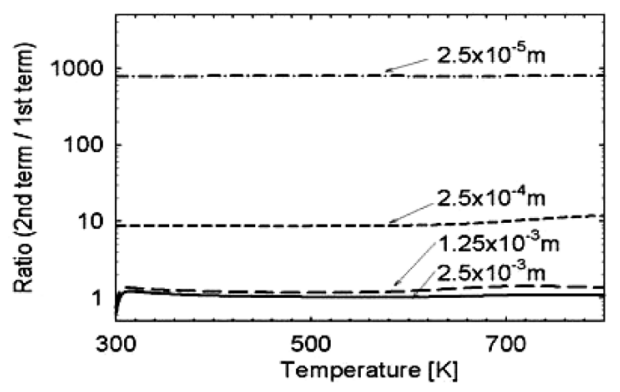

(a)

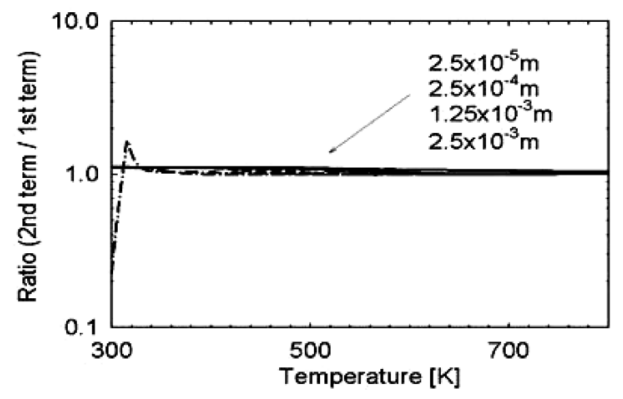

(b)

Fig. 8. Ratio of the second term to the first term of R.H.S. in Eq. (2): (a) Model I, and (b) Model III. 


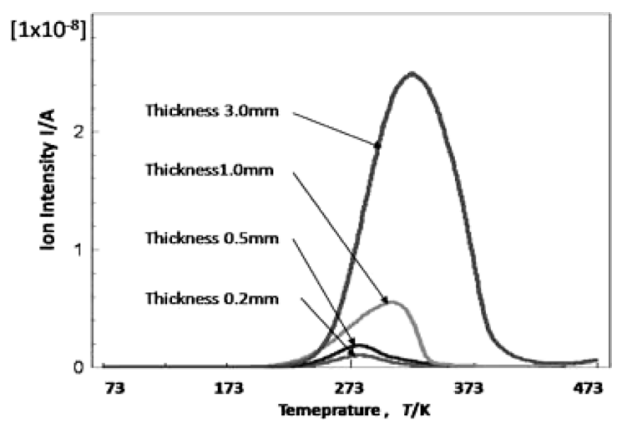

Fig. 9. The experimental desorption spectra for pure iron as a function of the specimen size. ${ }^{18)}$

term, $p_{0} \cdot \exp \left\{\left(-Q_{d}-E_{d}\right) / R T\right\} \cdot \theta$. The fact, that this term depends only on temperature with the other variables fixed, explains the inhibition of the shifting process of the desorption profile around $600 \mathrm{~K}$. This explanation also applies to the case of decreasing trap site density. When $N_{d}$ is small, namely the capacity of the trap site is small, the occupation ratio $\theta_{d}$ is close to unity if we assume the amount of charged hydrogen is constant, which makes the second term dominant just as in the first case. The appearance of the new peak at the beginning of heating in Fig. 6 results from the excess hydrogen which cannot be captured by the trap site.

Recently, this phenomenon, i.e. the peak profile remains stationary with the decrease of the specimen size, has been confirmed by the thermal desorption experiment using a pure iron specimen annealed at $1173 \mathrm{~K},{ }^{18)}$ where the plate specimen is used due to the difficulty of fabricating the very thin cylindrical specimen. The dimension of the specimen is $w \times 10 \mathrm{~mm} \times 10 \mathrm{~mm}$, where the thickness $w$ is changed from 3.0 to $0.2 \mathrm{~mm}$. The hydrogen desorption spectra are shown in Fig. 9. Since the pure iron specimen is fabricated by cold rolling and then annealed, the predominant defects, generated and remained in the specimen, are considered to be dislocations as in the case for the cylindrical specimen. The specimen thickness is less than $3.0 \mathrm{~mm}$ and the heating rate is $60 \mathrm{~K} / \mathrm{h}$ in this experiment. Thus the peak of the profile for the plate specimen is located in the lower temperature region compared with that for the cylindrical specimen in Fig. 3(a).

It is seen in Fig. 9 that the desorption peak shifts to the lower temperature as the specimen thickness decreases. With the specimen thickness less than $0.5 \mathrm{~mm}$, the position of the peak remains almost stationary at around $273 \mathrm{~K}$. The behavior of this peak shifting process in experiment corresponds well to that simulated by Model I, although the positions of the peak are remote from each other due to the difference in specimen size and experimental condition. Since other models explicitly incorporating the local equilibrium hypothesis cannot reproduce this trend, we hereafter concentrate on analyzing Model I in detail in terms of the reproduction of the experimental thermal desorption spectra.

\subsection{Numerical Reproduction of the Experimental Des- orption Spectra}

In Sec. 3, we found that the experimental desorption spectra, or the profiles derived by the non-diffusion model,

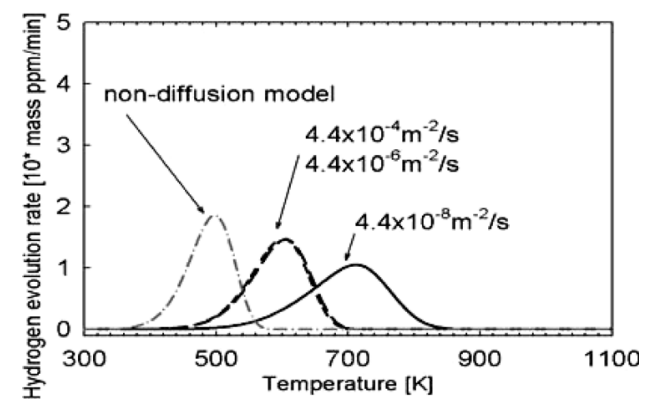

Fig. 10. Thermal desorption spectra simulated by Model I as a function of the pre-exponential factor $D_{0}$ of the diffusion coefficient.

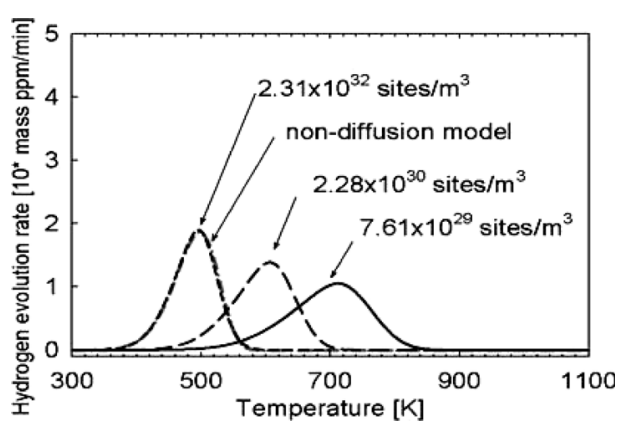

Fig. 11. Thermal desorption spectra simulated by Model I as a function of $k_{0} / p_{0}$.

cannot be reproduced just by changing relevant parameters contained in Model I. The experimental condition requires such parameters as the heating rate and the specimen size be fixed. The other parameters that should come under close scrutiny are the pre-exponential factor $D_{0}$ of the diffusion coefficient, the releasing rate $p_{0}$, and the capturing rate $k_{0}$ of the trap site.

Figure 10 shows that the profile simulated by Model I shifts to the lower temperature by increasing $D_{0}$ and is arrested at around $600 \mathrm{~K}$ over the value of approximately $10^{-6} \mathrm{~m}^{2} / \mathrm{s}$. The behavior of this peak shifting process is almost the same as those found in Figs. 6 and 7 due to the depletion of hydrogen in the interstitial lattice sites with the increase of $D_{0}$. Moreover, the value of $D_{0}$, determined by several experiments ${ }^{19,20)}$ and the theoretical calculation in the ideal bcc lattice, ${ }^{21)}$ is in the range of $10^{-8} \mathrm{~m}^{2} / \mathrm{s}-10^{-7}$ $\mathrm{m}^{2} / \mathrm{s}$, and, thus, $D_{0}$ is unsuitable for a fitting parameter.

Model I by Turnbull et al. ${ }^{13)}$ employed the relation of $p_{0} / k_{0}=N_{I}$. This relation is derived in the case of the perfect crystal lattice by imposing the local equilibrium hypothesis. In other words, in the simulation condition satisfying the local equilibrium, the hydrogen equilibrium in the perfect crystal lattice can be reached due to this relation. If we relax this relation, we can successfully obtain the experimental profile by adjusting the value of $p_{0} / k_{0}$, as shown in Fig. 11. The fitted value is found to be larger than $N_{I}=7.61 \times 10^{29} / \mathrm{m}^{3}$ that is estimated for the bcc Fe lattice. This can be understood from the fact that the lattice containing the interstitial sites is distorted due to the introduction of defects, such as a dislocation. Thus, in general, we suggest that the values of $p_{0}$ and $k_{0}$ be determined independently. 


\subsection{Fundamental Numerical Model for Thermal Des- orption Analysis}

It is found in the previous discussion that Model I has the best potential for becoming a fundamental model of thermal desorption spectra on the condition that the relation of $p_{0} / k_{0}=N_{I}$ is relaxed. This indicates clearly that the local equilibrium hypothesis does not hold generally for the thermal desorption process, in particular, for the thin specimen limit. In the following, Model I without the constraint relation of $p_{0} / k_{0}=N_{I}$ is called modified Model I. We can consider this a fundamental model for TDA. For comparison, below, we discuss more the other models which could reproduce the experimental spectra.

Let us consider the reason why the non-diffusion model is successful in reproducing the experimental profile. This model neglects the diffusion process on the assumption of fast hydrogen diffusion and to impose the equilibrium hypothesis; hydrogen in the interstitial lattice site is made to be depleted and to be desorbed at each instant in the process of rising temperature. In the realistic situation, when hydrogen in the interstitial lattice site is depleted, as mentioned above, the peak position is determined only by the releasing process. Thus the peak in the non-diffusion model should appear at around $600 \mathrm{~K}$ as shown in Fig. 10. In the non-diffusion model, however, the peak appears at lower temperature because of the excess hydrogen released from the trap site to compensate the depletion of hydrogen in the interstitial lattice site due to the constraint of the equilibrium hypothesis. This is the reason why the non-diffusion model could reproduce the experimental profile. However, from this discussion, it is predicted that the trap site density is overestimated in the non-diffusion model.

In the case of Model II, the desorption peak is determined by the effective diffusion coefficient which is a function of the trap site density. The fact that the trap site density can be made small without limit enables this model to reproduce the experimental profile, but the trap site density is underestimated. Moreover, since this model does not include the process of capturing and releasing hydrogen, it is not possible to simulate more than a peak in the profile.

According to the above discussion, the modified Model I is fundamental to simulate the hydrogen desorption spectra within the broad range of parameters in order to identify the hydrogen state in steels. Recently, the atomistic calculations ${ }^{21,22)}$ are employed to evaluate binding and activation energies. The simulation of the desorption spectra is performed by the numerical model employing the parameters which are evaluated by the atomistic calculation. ${ }^{23)}$ The objective of these works is to narrow down the range of unknown simulation parameters. Accordingly, on the basis of the modified Model I, we believe that the efforts should be devoted to identify unknown parameters theoretically and experimentally such as $k_{0}, p_{0}, D_{0}$, and $E_{i}$, which depend on the microstructure of specimens. In addition, recent development of the low-temperature thermal desorption experiment $^{3)}$ makes it possible to determine and validate parameters.

\section{Conclusion}

We performed a comparative study on three numerical models to search for the best model to describe correctly the experimental hydrogen thermal desorption spectra. The sensitivity of various parameters in the models to the behavior of thermal desorption spectra is systematically examined in detail. The focus is on the local equilibrium hypothesis since this hypothesis, which is contained in most of the previous models, affects and limits the behavior of thermal desorption profile. The validity of the hypothesis is finally checked by the experimental result for pure iron. We found that the local equilibrium hypothesis does not hold generally in the thermal desorption process, in particular, for the thin specimen, and the mass conservation equation of hydrogen with trap sites in activation energy form constitutes the fundamental model for describing thermal desorption spectra.

\section{Acknowledgements}

This study was carried out as a part of research activities of "Fundamental Studies on Technologies for Steel Materials with Enhanced Strength and Functions" by Consortium of JRCM (The Japan Research and Development Center of Metals). Financial support from NEDO (New Energy and Industrial Technology Development Organization) is gratefully acknowledged.

\section{REFERENCES}

1) K. Takai, G. Yamauchi, M. Nakamura and M. Nagumo: J. Jpn. Inst. Met., 62 (1998), 267.

2) M. Nagumo: Zairyo-to-Kankyo, 55 (2006), 42.

3) K. Fujita, Y. Sato, H. Suzuki, K. Takai and Y. Hagihara: CAMP-ISIJ, 21 (2008), 1376, CD-ROM.

4) A. McNabb and P. K. Foster: Trans. Metall. Soc. ASME, 227 (1963), 618.

5) R. A. Oriani: Acta Metall., 18 (1970), 147.

6) G. M. Pressouyre and I. M. Bernstein: Metall. Trans. A, 9A (1978), 1571.

7) K. L. Wilson and M. I. Baskes: J. Nucl. Mater, 76-77 (1978), 291

8) M. Iino: Metall. Trans. A, 18A (1987), 1559.

9) W. Y. Choo and J. Y. Lee: Metall. Trans. A, 13A (1982), 135.

10) J. Abrefah, D. R. Olander and M. Balooch: J. Appl. Phys., 67 (1990), 3302 .

11) K. Ono and M. Meshii: Acta Metall. Mater., 40 (1992), 1357.

12) E. Abramov and D. Eliezer: Metall. Mater. Trans. A, 25A (1994), 951.

13) A. Turnbull, R. B. Hutchings and D. H. Ferriss: Mater. Sci. Eng. A, 238A (1997), 317.

14) M. Enomoto, D. Hirakami and T. Tarui: ISIJ Int., 46 (2006), 1381.

15) T. Yamaguchi and M. Nagumo: ISIJ Int., 43 (2003), 514.

16) K. Ebihara, T. Suzudo, H. Kaburaki, K. Takai and S. Takebayashi: ISIJ Int., 47 (2007), 1131.

17) K. Ebihara, T. Suzudo, H. Kaburaki, K. Takai and S. Takebayashi: Tetsu-to-Hagané, 94 (2008), 522.

18) Y. Sato, K. Fujita, H. Suzuki, K. Takai, Y. Hagihara and N. Ishikawa: CAMP-ISIJ, 22 (2009), 559.

19) Eds. B. Bokstein and N. Balandina: Hydrogen Diffusion in Metals A 30-Year Retrospective, ed. by D. J. Fisher, Scitec Publications, (1999), 16

20) H. Hagi, Y. Hayashi and N. Ohtani: Trans. Jpn. Inst. Met., 20 (1979), 349.

21) D. E. Jiang and E. A. Carter: Phys. Rev., B70 (2004), 064102.

22) M. Yamaguchi, K. Ebihara, T. Suzudo, M. Itakura and H. Kaburaki: Proceedings of JSME 21th Computational Mechanics Conference (2008), 35, CD-ROM.

23) K. Ebihara, M. Yamaguchi, T. Suzudo, H. Kaburaki and K. Takai: CAMP-ISIJ, 20 (2007), 1259. 